\title{
Thermally Induced Asymmetric Buckling of Circular Monolayer Graphene
}

\author{
Haw-Long Lee and Win-Jin Chang \\ Department of Mechanical Engineering, Kun Shan University, Tainan 71003, Taiwan \\ Correspondence should be addressed to Win-Jin Chang; changwj@mail.ksu.edu.tw
}

Received 25 June 2013; Revised 4 August 2013; Accepted 7 August 2013

Academic Editor: Sheng-Rui Jian

Copyright ( 2013 H.-L. Lee and W.-J. Chang. This is an open access article distributed under the Creative Commons Attribution License, which permits unrestricted use, distribution, and reproduction in any medium, provided the original work is properly cited.

The asymmetric buckling behaviors of circular monolayer graphene with clamped boundary condition subjected to temperature change are analytically studied based on the nonlocal elasticity theory, including the small length effect. The axisymmetrical and asymmetric critical buckling temperatures and mode shape of different order modes are obtained. According to the analysis, the asymmetric critical buckling temperature of monolayer graphene is larger than the axisymmetric one. The axisymmetrical and asymmetric critical buckling temperatures decrease with increasing nonlocal parameter. In addition, nodal diametrical lines and nodal circles can be found from the modal shapes. In order to avoid destruction of the sensors due to buckling of the structure, they can be placed at the nodal diametrical lines or nodal circles.

\section{Introduction}

Graphene has recently attracted significant research interest due to its potential applications in nanobiological devices and nanoelectromechanical systems such as single-electron transistor [1], actuators [2], and photodetectors [3]. Because graphene is only one atom layer thick, it is easily amenable to external influences, including thermal loading and mechanical deformation [4-8]. The external mechanical loading can affect its electronic structure and physical properties. For example, there were significant changes in graphene's band structure for uniaxial strains of up to $15 \%$ [5]. The magnetic behavior of multilayer graphene can be affected by mechanical transverse deformation [7].

In the last years, continuum theory has received the attention of many researchers for analysis of nanostructures. This is because available experiments on nanoscale materials are difficult to perform, and molecular dynamic simulations are highly time-consuming. Accordingly, various size-dependent continuum theories such as strain gradient theory [9], couple stress theory [10], and nonlocal elasticity theory [11] have been proposed. Among these theories, nonlocal elasticity theory including the small-scale effect has often been used to study the buckling and vibration responses of nanostructures [12-16]. The theory was initiated by Eringen [17] in the early 1970 s and stated that the stresses at a point depend on strains at all the points of the body.

This paper focuses on the buckling behavior of graphene. In recent years, some researchers have studied the buckling of graphene by using different methods. For example, Frank et al. [18] experimentally studied the compression buckling strain of a graphene sheet. Neek-Amal and Peeters [19] investigated the stability of circular monolayer graphene subjected to a radial load using molecular dynamics simulations. Natsuki et al. [20] studied the buckling properties of circular double-layered graphene sheets using plate theory and found that buckling stability is significantly affected by the buckling mode shapes.

In this paper, thermally induced asymmetric buckling of circular monolayer graphene is studied based on the nonlocal elasticity theory by using the analytical method. According to the analysis, the axisymmetrical and asymmetric buckling temperatures and mode shape for the graphene with various modes are obtained. In addition, nodal diametrical lines and nodal circles in the buckling mode shape are also investigated. 


\section{Physical Model and Mathematical Formulation}

2.1. Governing Equation and Boundary Conditions. Consider that a circular graphene sheet is clamped on its edge, which is modeled as a clamped circular plate with the radius $R$ and thickness $h$ subjected to temperature change $T$ as depicted in Figure 1. Based on the nonlocal elasticity theory, the nonlocal constitutive relation of circular plate under the thermal load is given by [21]

$$
D \nabla^{4} w(r, \theta)+\left[1-\left(e_{0} a\right)^{2} \nabla^{2}\right] N_{T} \nabla^{2} w(r, \theta)=0,
$$

where $w$ is the displacement along the thickness of the graphene. $r$ and $\theta$ are the radial and circumferential coordinates. $\nabla^{2}=\left(\left(\partial^{2} / \partial r^{2}\right)+(1 / r)(\partial / \partial r)+\left(1 / r^{2}\right)\left(\partial^{2} / \partial \theta^{2}\right)\right)$ is the Laplacian operator in polar coordinates. $e_{0} a$ is the nonlocal parameter revealing the nanoscale size effect. $N_{T}$ and $D$ are the thermal buckling load and flexural bending rigidity of graphene, respectively. They are

$$
\begin{gathered}
N_{T}=\frac{E \alpha T}{1-\mu} h, \\
D=\frac{E h^{3}}{12\left(1-\mu^{2}\right)},
\end{gathered}
$$

where $E, \mu$, and $\alpha$ are Young's modulus, Poisson's ratio, and thermal expansion coefficient of graphene, respectively.

The corresponding boundary conditions are

$$
w(R)=0, \quad \frac{\partial w(R)}{\partial r}=0 .
$$

The nondimensional variables are introduced and defined as follows:

$$
\begin{gathered}
\xi=\frac{r}{R}, \quad \bar{w}=\frac{w}{R}, \\
\varepsilon=\frac{e_{0} a}{R}, \quad T^{*}=\frac{12 \alpha T R^{2}}{(1+\mu) h^{2}},
\end{gathered}
$$

where $\xi, \bar{w}, \varepsilon$, and $T^{*}$ denote the nondimensional radius, outplane displacement, nonlocal parameter, and buckling temperature.

Using the dimensionless variables given by (4), the governing equation and associated boundary conditions can be simplified to the following dimensionless form:

$$
\begin{gathered}
\bar{\nabla}^{2}\left(\bar{\nabla}^{2} \bar{w}+\lambda^{2} \bar{w}\right)=0, \\
\bar{w}(1)=0, \quad \frac{\partial \bar{w}(1)}{\partial \xi}=0,
\end{gathered}
$$

where

$$
\begin{gathered}
\bar{\nabla}^{2}=\left(\frac{\partial^{2}}{\partial \xi^{2}}+\frac{\partial}{\xi \partial \xi}+\frac{\partial^{2}}{\xi^{2} \partial \theta^{2}}\right), \\
\lambda^{2}=\frac{T^{*}}{1-\varepsilon^{2} T^{*}} .
\end{gathered}
$$

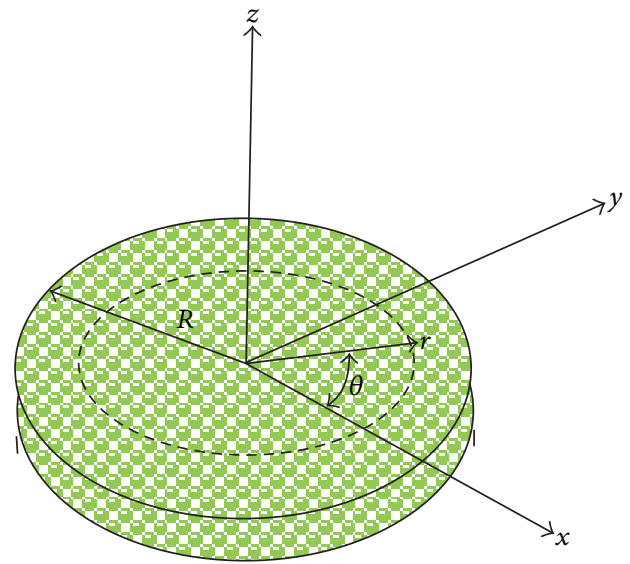

FIGURE 1: A clamped circular graphene sheet with the radius $R$ under temperature change $T$.

2.2. Asymmetric Buckling Mode. It is well known that the normal vibration of an elastic linear system is harmonic. The displacement of monolayer graphene for a harmonic vibration can therefore be separated as follows:

$$
\bar{w}(\xi, \theta)=f(\xi) \cos n \theta, \quad n=0,1,2,3, \ldots,
$$

where $n$ is the number of nodal diametrical lines.

Substituting the relation of (9) into (5), we have

$$
\left(\frac{d^{2}}{d \xi^{2}}+\frac{d}{\xi d \xi}-\frac{n^{2}}{\xi^{2}}\right)\left[\frac{d^{2}}{d \xi^{2}}+\frac{d}{\xi d \xi}+\left(\lambda^{2}-\frac{n^{2}}{\xi^{2}}\right)\right] f(\xi)=0 .
$$

The general solution of the earlier equation should be expressed as

$$
\begin{aligned}
f(\xi)= & A_{1} \xi^{n}+A_{2} \xi^{-n}+A_{3} J_{n}(\lambda \xi) \\
& +A_{4} Y_{n}(\lambda \xi), \quad n=1,2,3
\end{aligned}
$$

where $J_{n}$ and $Y_{n}$ are the Bessel functions of the first and second kinds of order $n . A_{1}, \ldots, A_{4}$ are arbitrary constants depending on the boundary conditions.

Substituting the boundary conditions given by (6) into (11), we can obtain the characteristic functions as

$$
J_{n+1}\left(\lambda_{m n}\right)=0
$$

where $\lambda_{m n}$ are the roots of the Bessel function of the first kind of order $n+1$ for mode $m$.

Once the parameter $\lambda_{m n}$ is given, the following nondimensional buckling temperature can be obtained from (8):

$$
T_{m n}^{*}=\frac{\lambda_{m n}^{2}}{1+\varepsilon^{2} \lambda_{m n}^{2}} .
$$

In addition, the nondimensional buckling mode shape can be expressed as

$$
\bar{w}_{m n}(\xi, \theta)=A_{m n} J_{n}\left(\lambda_{m n} \xi\right) \cos n \theta .
$$


There are $n$ nodal diametrical lines, which are straight lines with no curve and no displacement. In addition, there are $m$ nodal circles, which are no displacement. The radius of nodal circle $\rho$ can be solved by

$$
J_{n}\left(\lambda_{m n} \rho\right)=0 .
$$

2.3. Axisymmetric Buckling Mode. For the axisymmetric buckling mode (i.e., $n=0$ ), using (10), the differential equation can be expressed as

$$
\left(\frac{d^{2}}{d \xi^{2}}+\frac{d}{\xi d \xi}\right)\left(\frac{d^{2}}{d \xi^{2}}+\frac{d}{\xi d \xi}+\lambda^{2}\right) f(\xi)=0
$$

The general solution can be written as

$$
f_{s}(\xi)=B_{1}+B_{2} \ln \xi+B_{3} J_{0}(\lambda \xi)+B_{4} Y_{0}(\lambda \xi),
$$

where $J_{0}$ and $Y_{0}$ are the Bessel functions of the first and second kinds of order zero and $B_{1}, \ldots, B_{4}$ are the arbitrary constants.

Applying the boundary conditions, the characteristic function, $J_{1}\left(\lambda_{m 0}\right)=0$, can be obtained. The result is the same as that derived by Farajpour et al. [21].

Using (14)-(15) and setting $n=0$, the nondimensional buckling temperature, buckling shape, and radii of nodal circles of axisymmetric monolayer graphene can also be determined. It is noted that there are no nodal diametrical lines, but there are $m$ nodal circles for axisymmetric buckling mode.

\section{Results and Discussion}

To study the effect of small length scale on the buckling behaviors of circular monolayer graphene, critical buckling temperature and mode shape are analyzed for different mode numbers and nonlocal parameters. Figure 2 shows the nondimensional critical buckling temperature as a function of nonlocal parameters for asymmetric monolayer graphene with clamped boundary conditions with $n=1$ for the first five modes. The parameter value of $e_{0} a / R=0$ implies that the nonlocal effect is neglected. It can be seen that the effect of nonlocal parameter on the critical buckling temperature is significant, especially at higher-order modes. The critical buckling temperature decreases with increasing nonlocal parameter. This is because the internal interaction force increases as the nonlocal parameter increases.

Figure 3 depicts the critical buckling temperature ratio for asymmetric (i.e., $n=1$ ) and axisymmetric (i.e., $n=0$ ) monolayer graphene at different modes. The critical buckling temperature of asymmetric monolayer graphene is larger than that of axisymmetric ones. The discrepancy is larger for a lower-order mode. Figures 4(a) and 4(b) illustrate the axisymmetric (i.e., $n=0$ ) buckling mode shape of circular monolayer graphene with $e_{0} a / R=0.05$ for $m=1$ and $m=2$, respectively. In practical applications, the lower buckling modes are easier to reach than the higher-order modes. Therefore, the parameters $m=1$ and $m=2$ are selected in the analysis. The nodal circles $r=$ const can be seen for the figures. There are $m$ circular nodes for different values of $m$.

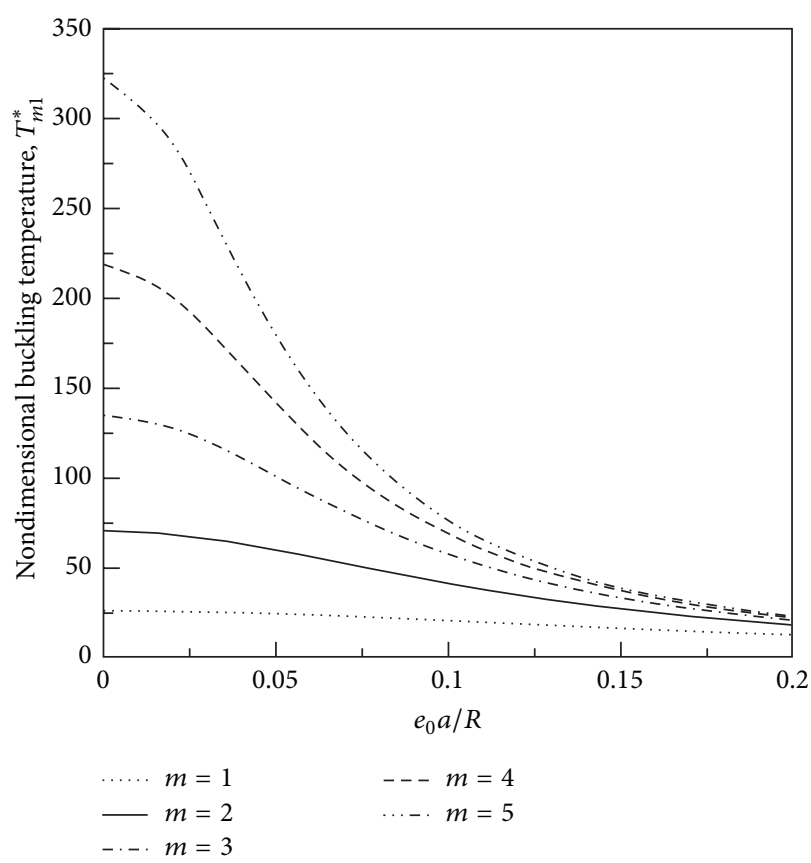

FIGURE 2: The critical buckling temperature as a function of nonlocal parameter for asymmetric monolayer graphene with $n=1$.

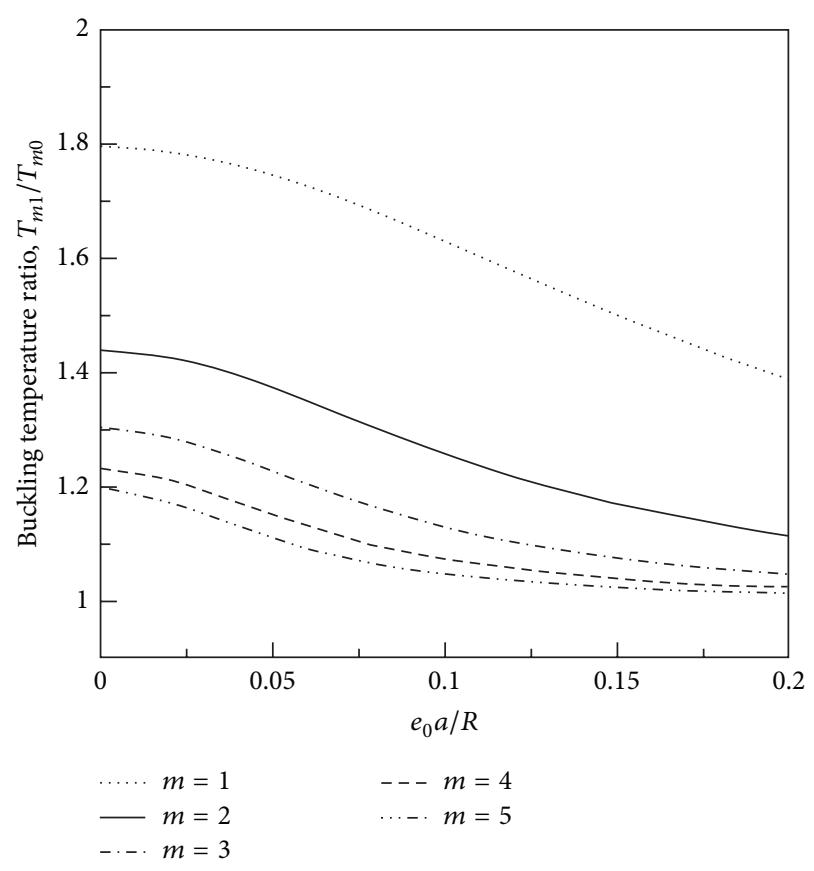

FIGURE 3: The critical buckling temperature ratio for asymmetric and axisymmetric monolayer graphene.

According to the calculation, the buckling temperatures are $T_{10}^{*}=14.16$ and $T_{20}^{*}=43.86$ for $m=1$ and $m=2$, respectively.

Figure 5 shows the asymmetric buckling mode shape and nodal lines of circular monolayer graphene with $e_{0} a / R=0.05$ for different values of $m$ and $n$. The nodal lines are straight 


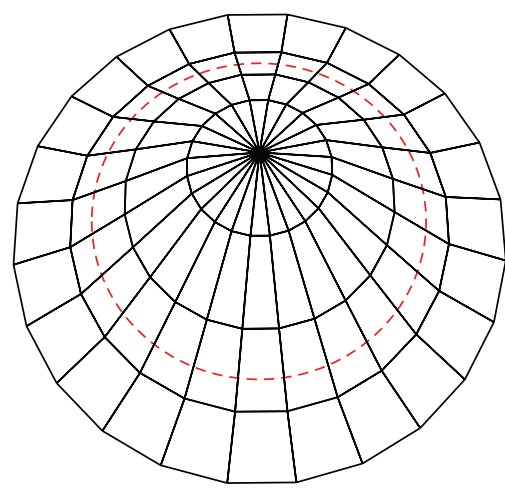

- - - Nodal circle

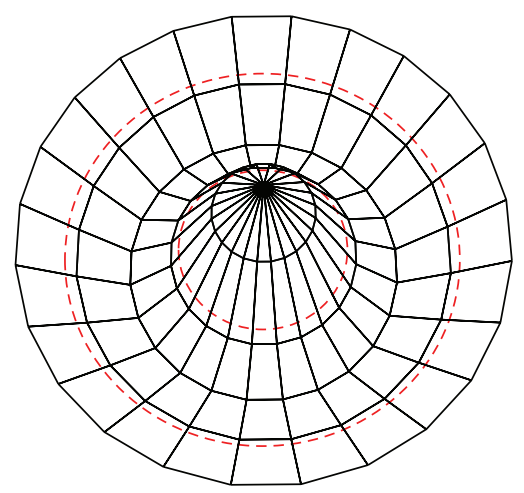

- - - Nodal circle

(a)

(b)

FIgURE 4: Axisymmetric buckling mode shape of circular monolayer graphene with $e_{0} a / R=0.05$ for (a) $m=1$ and (b) $m=2$.

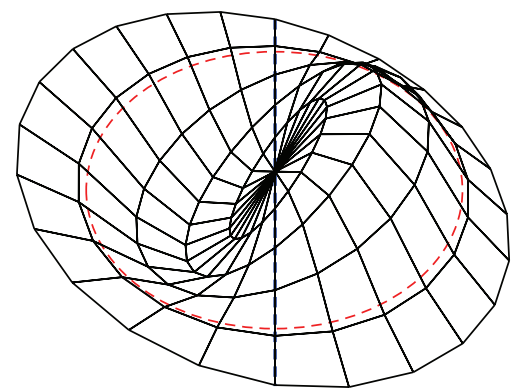

- - - Nodal circle

- - - Nodal diametrical line

(a)

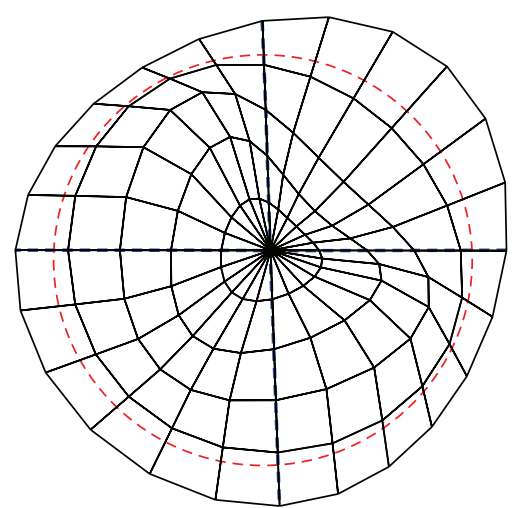

- - Nodal circle

- - - Nodal diametrical line

(c)

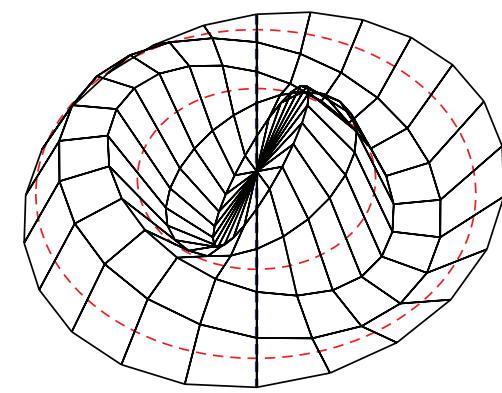

- - Nodal circle

- - Nodal diametrical line

(b)

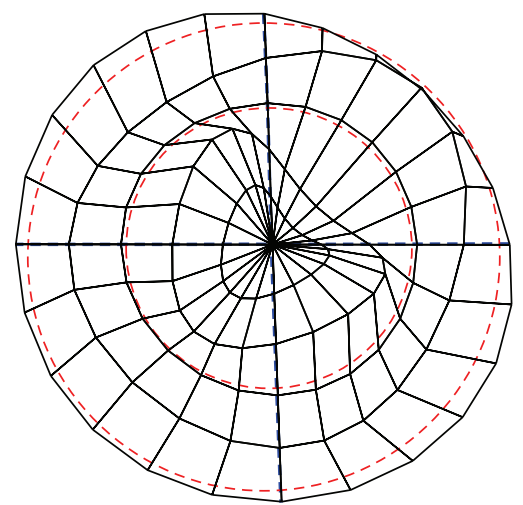

- - Nodal circle

- - - Nodal diametrical line

(d)

Figure 5: Asymmetric buckling mode shape of circular monolayer graphene with $e_{0} a / R=0.05$ for (a) $m=1, n=1$, (b) $m=2, n=1$, (c) $m=1, n=2$, and (d) $m=2, n=2$.

diametrical lines $\theta=$ const and circles $r=$ const. There are $m$ nodal circulars and $n$ straight diametrical lines for the case analyzed in the figure. The sensors used to measure modal data can be placed at the nodal diametrical lines and nodal circles in order to avoid destruction of the sensors due to structure buckling. The buckling temperatures of $T_{11}^{*}=24.73$ for $m=1$ and $n=1, T_{21}^{*}=60.19$ for $m=2$ and $n=1$, $T_{12}^{*}=36.95$ for $m=1$ and $n=2$, and $T_{22}^{*}=76.9$ for $n=2$ and $m=2$ are obtained from this calculation.

Figure 6 depicts the nondimensional radius of nodal circle of mode 1 as a function of nonlocal parameter. It can be seen that the radius of nodal circle obviously increases 


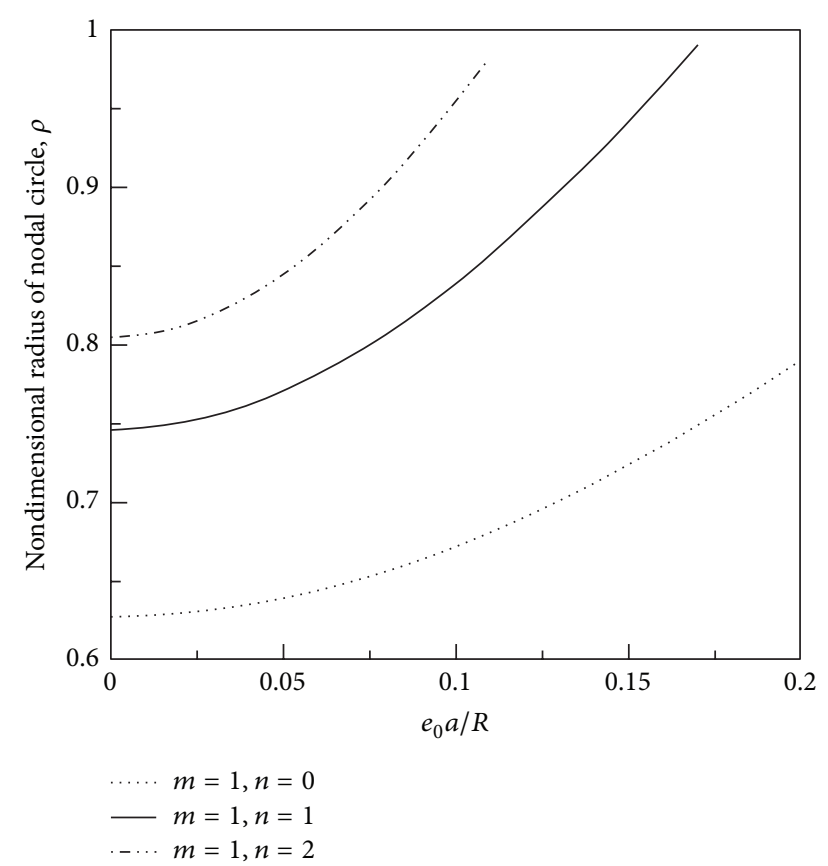

FIgURE 6: Nondimensional radius of nodal circle as a function of nonlocal parameter.

TABLE 1: Nondimensional radii of nodal circles as a function of nonlocal parameter with $m=2$.

\begin{tabular}{|c|c|c|c|c|c|c|}
\hline \multirow{2}{*}{$e_{0} a / R$} & \multicolumn{2}{|c|}{$n=0$} & \multicolumn{2}{|c|}{$n=1$} & \multicolumn{2}{|c|}{$n=2$} \\
\hline & $S$ & $L$ & $S$ & $L$ & $S$ & $L$ \\
\hline 0 & 0.343 & 0.787 & 0.455 & 0.833 & 0.526 & 0.862 \\
\hline 0.01 & 0.344 & 0.789 & 0.457 & 0.836 & 0.529 & 0.866 \\
\hline 0.02 & 0.346 & 0.795 & 0.462 & 0.845 & 0.536 & 0.879 \\
\hline 0.03 & 0.350 & 0.804 & 0.470 & 0.860 & 0.548 & 0.899 \\
\hline 0.04 & 0.356 & 0.817 & 0.480 & 0.879 & 0.565 & 0.926 \\
\hline 0.05 & 0.363 & 0.834 & 0.494 & 0.904 & 0.585 & 0.960 \\
\hline 0.06 & 0.372 & 0.854 & 0.510 & 0.934 & 0.610 & 0.999 \\
\hline 0.07 & 0.382 & 0.877 & 0.528 & 0.967 & 0.637 & \\
\hline 0.08 & 0.393 & 0.902 & 0.549 & & 0.668 & \\
\hline 0.09 & 0.405 & 0.931 & 0.571 & & 0.700 & \\
\hline 0.1 & 0.419 & & 0.595 & & 0.735 & \\
\hline 0.11 & 0.433 & & 0.620 & & 0.772 & \\
\hline 0.12 & 0.448 & & 0.647 & & 0.810 & \\
\hline 0.13 & 0.464 & & 0.675 & & 0.850 & \\
\hline 0.14 & 0.480 & & 0.704 & & 0.891 & \\
\hline 0.15 & 0.498 & & 0.733 & & 0.933 & \\
\hline 0.16 & 0.515 & & 0.764 & & & \\
\hline 0.17 & 0.534 & & 0.795 & & & \\
\hline 0.18 & 0.552 & & 0.826 & & & \\
\hline 0.19 & 0.571 & & 0.859 & & & \\
\hline 0.2 & 0.591 & & 0.891 & & & \\
\hline
\end{tabular}

$S$ : radius of smaller nodal circle.

$L$ : radius of lager nodal circle.

with increasing the nonlocal parameter. The nondimensional radii of nodal circles as a function of nonlocal parameter with $m=2$ are listed in Table 1 . There are two nodal circles for the case of $m=2$. The radii of the two nodal circles increase with increasing the value of $n$ and nonlocal parameter. The large one can easily exceed the range of the graphene when the nonlocal parameter is too large. For the case of larger $n$ and nonlocal parameter, both circles may be out of the range.

\section{Conclusions}

The effect of the small length scale on the critical buckling temperature and mode shape of circular monolayer graphene with clamped boundary condition was analyzed using the nonlocal elasticity theory. The results showed that the critical buckling temperature decreased with increasing nonlocal parameter. The critical buckling temperature of asymmetric monolayer graphene was larger than that of axisymmetric one. It can be observed that there were $m$ nodal circulars and $n$ straight diametrical lines for the asymmetric buckling of circular monolayer graphene. In addition, the radii of nodal circles obviously increased with increasing the nonlocal parameter.

\section{Acknowledgment}

The authors wish to thank the National Science Council of the Republic of China in Taiwan for providing financial support for this study under Projects NSC 101-2221-E-168-006 and NSC 101-2221-E-168 -009.

\section{References}

[1] C. Stampfer, E. Schurtenberger, F. Molitor, J. Güttinger, T. Ihn, and K. Ensslin, "Tunable graphene single electron transistor," Nano Letters, vol. 8, no. 8, pp. 2378-2383, 2008.

[2] S. Park, J. An, J. W. Suk, and R. S. Ruoff, "Graphene-based actuators," Small, vol. 6, no. 2, pp. 210-212, 2010.

[3] T. Mueller, F. Xia, and P. Avouris, "Graphene photodetectors for high-speed optical communications," Nature Photonics, vol. 4, no. 5, pp. 297-301, 2010.

[4] W. Bao, F. Miao, Z. Chen et al., "Controlled ripple texturing of suspended graphene and ultrathin graphite membranes," Nature Nanotechnology, vol. 4, no. 9, pp. 562-566, 2009.

[5] F. Guinea, M. I. Katsnelson, and A. K. Geim, "Energy gaps and a zero-field quantum hall effect in graphene by strain engineering," Nature Physics, vol. 6, no. 1, pp. 30-33, 2010.

[6] V. M. Pereira, A. H. Castro Neto, and N. M. R. Peres, "Tightbinding approach to uniaxial strain in graphene," Physical Review B, vol. 80, no. 4, Article ID 045401, 2009.

[7] E. V. Castro, M. P. López-Sancho, and M. A. H. Vozmediano, "Effect of pressure on the magnetism of bilayer graphene," Physical Review B, vol. 84, no. 7, Article ID 075432, 2011.

[8] H. S. Shen, L. Shen, and C. L. Zhang, "Nonlocal plate model for nonlinear bending of single-layer graphene sheets subjected to transverse loads in thermal environments," Applied Physics A, vol. 103, no. 1, pp. 103-112, 2011.

[9] D. C. C. Lam, F. Yang, A. C. M. Chong, J. Wang, and P. Tong, "Experiments and theory in strain gradient elasticity," Journal of the Mechanics and Physics of Solids, vol. 51, no. 8, pp. 1477$1508,2003$. 
[10] F. Yang, A. C. M. Chong, D. C. C. Lam, and P. Tong, "Couple stress based strain gradient theory for elasticity," International Journal of Solids and Structures, vol. 39, no. 10, pp. 2731-2743, 2002.

[11] A. C. Eringen, Nonlocal Continuum Field Theories, Springer, New York, NY, USA, 2002.

[12] H. L. Lee and W. J. Chang, "Vibration analysis of fluid-conveying double-walled carbon nanotubes based on nonlocal elastic theory," Journal of Physics Condensed Matter, vol. 21, no. 11, Article ID 115302, 2009.

[13] W. J. Chang and H. L. Lee, "Buckling temperature of a singlewalled carbon nanotube using nonlocal Timoshenko beam model," Journal of Computational and Theoretical Nanoscience, vol. 7, no. 11, pp. 2367-2371, 2010.

[14] H. L. Lee, J. C. Hsu, and W. J. Chang, "Frequency shift of carbonnanotube-based mass sensor using nonlocal elasticity theory," Nanoscale Research Letters, vol. 5, no. 11, pp. 1774-1778, 2010.

[15] H. L. Lee and W. J. Chang, "Dynamic modelling of a singlewalled carbon nanotube for nanoparticle delivery," Proceedings of the Royal Society A, vol. 467, no. 2127, pp. 860-868, 2011.

[16] H. L. Lee and W. J. Chang, "Vibration analysis of viscoelastic carbon nanotubes," Micro \& Nano Letters, vol. 7, no. 12, pp. 1308-1316, 2012.

[17] A. C. Eringen, "Linear theory of nonlocal elasticity and dispersion of plane waves," International Journal of Engineering Science, vol. 10, no. 5, pp. 425-435, 1972.

[18] O. Frank, G. Tsoukleri, J. Parthenios et al., "Compression behavior of single-layer graphenes," ACS Nano, vol. 4, no. 6, pp. 3131-3138, 2010.

[19] M. Neek-Amal and F. M. Peeters, "Buckled circular monolayer graphene: a graphene nano-bowl," Journal of Physics Condensed Matter, vol. 23, no. 4, Article ID 045002, 2011.

[20] T. Natsuki, J. Shi, and Q. Ni, "Buckling instability of circular double-layered graphene sheets," Journal of Physics Condensed Matter, vol. 24, no. 13, Article ID 135004, 2012.

[21] A. Farajpour, M. Mohammadi, A. R. Shahidi, and M. Mahzoon, "Axisymmetric buckling of the circular graphene sheets with the nonlocal continuum plate model," Physica E, vol. 43, no. 10, pp. 1820-1825, 2011. 

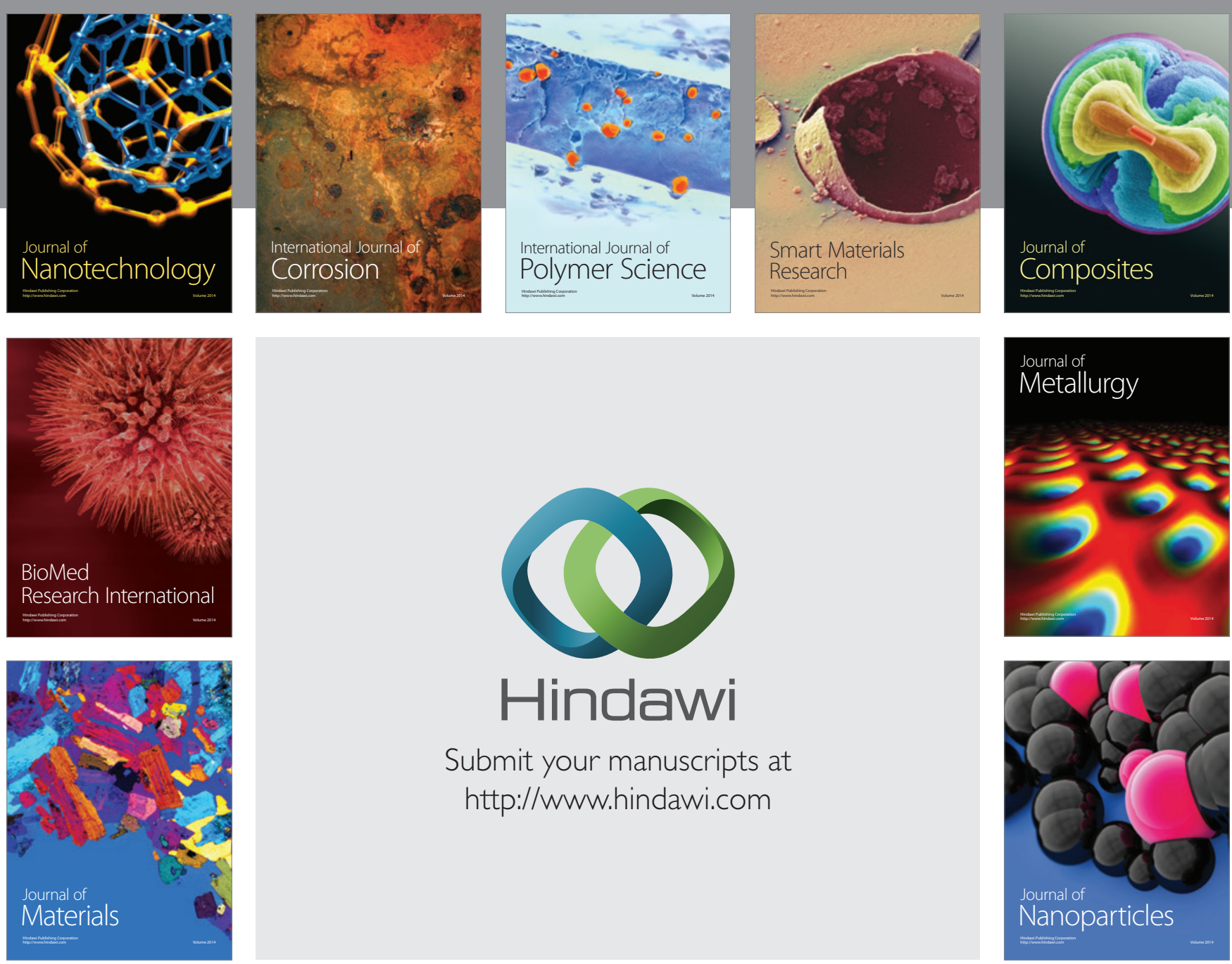

Submit your manuscripts at http://www.hindawi.com
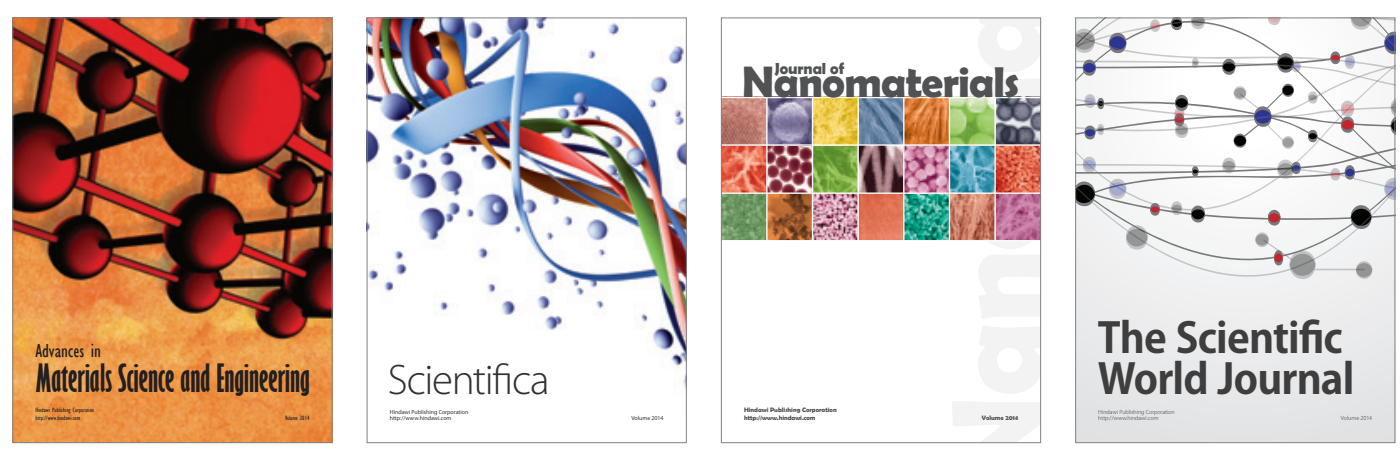

\section{The Scientific World Journal}
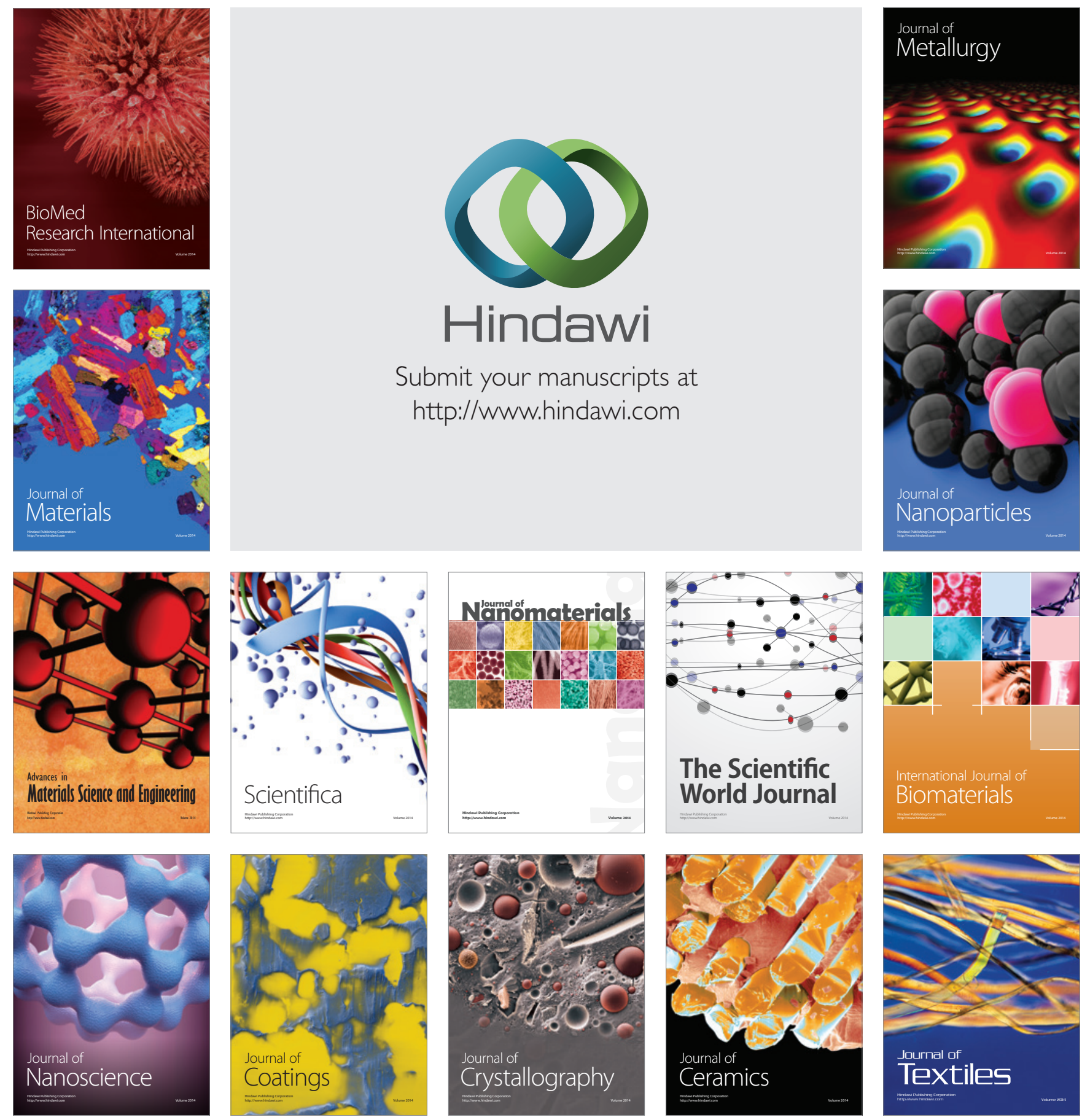